\title{
Genetic diversity among Pasteurella multocida strains of avian, bovine, ovine and porcine origin from England and Wales by comparative sequence analysis of the 16S rRNA gene
}

\author{
Robert L. Davies
}

Correspondence

Robert L. Davies

r.l.davies@bio.gla.ac.uk

Received 16 June 2004

Revised 23 August 2004

Accepted 20 September 2004
Division of Infection and Immunity, Institute of Biomedical and Life Sciences, Joseph Black Building, University of Glasgow, Glasgow G12 800, UK
Genetic diversity among 86 Pasteurella multocida isolates was investigated by comparative sequence analysis of a 1468 bp fragment of the 16S rRNA gene. The strains included 79 field isolates recovered from birds (poultry) (22), cattle (21), pigs (26) and sheep (10) within England and Wales, four Asian isolates associated with bovine haemorrhagic septicaemia, and the type strains of the three subspecies of $P$. multocida. Dulcitol and sorbitol fermentation patterns were also determined to establish correlations between subspecies status and phylogenetic relatedness. Nineteen $16 \mathrm{~S}$ rRNA types were identified, but these were clustered into two distinct phylogenetic lineages, $A$ and $B$. Sequences within lineages $A$ and $B$ had a mean number of nucleotide differences of $21 \cdot 12 \pm 3 \cdot 90$. Isolates within lineage $A$ were associated with birds, cattle, pigs and sheep, whereas those belonging to lineage $B$ were recovered from birds and a cat. Eighty-seven per cent of the isolates were classified as $P$. multocida subsp. multocida by dulcitol and sorbitol fermentation patterns, but these have diverse 16S rRNA gene sequences that were represented in both lineages $\mathrm{A}$ and $\mathrm{B}$. Avian $P$. multocida subsp. septica isolates were associated exclusively with lineage $\mathrm{B}$, but bovine $P$. multocida subsp. septica isolates were present in lineage A. P. multocida subsp. gallicida isolates of avian, bovine and porcine origin represent a homogeneous group within lineage A, but they have the same 16S rRNA type as certain $P$. multocida subsp. multocida isolates. These findings provide strong support for the view that dulcitol and sorbitol fermentation patterns are inaccurate indicators of genetic relatedness among $P$. multocida strains. Avian capsular type B isolates and capsular type B and $E$ isolates associated with haemorrhagic septicaemia of cattle and water buffaloes are closely related and form a distinct cluster within lineage $A$. The current subspecies nomenclature of $P$. multocida neither accurately reflects the $16 \mathrm{~S}$ rRNA-based phylogenetic relationships among isolates nor does it adequately encompass the full range of diversity within the species. The study provides a 16S rRNA-based evolutionary framework that will form the basis of further studies into the genetic diversity of $P$. multocida and will also help in the reclassification of the species.

\section{INTRODUCTION}

Pasteurella multocida represents a heterogeneous group of Gram-negative bacteria that are commensals in the upper respiratory tract of many mammals and birds (Rimler \& Rhoades, 1989). The organism is also a primary or secondary pathogen and is responsible for a wide range of economically important diseases in domesticated animals throughout the world. Infections caused by P. multocida include fowl cholera of poultry (Rhoades \& Rimler, 1989; Rimler \& Glisson, 1997), progressive atrophic rhinitis of

Abbreviations: MLEE, multilocus enzyme electrophoresis; OMP, outermembrane protein. pigs (Chanter \& Rutter, 1989), pneumonia of cattle, sheep and pigs (Chanter \& Rutter, 1989; Frank, 1989), and haemorrhagic septicaemia of cattle and water buffaloes in certain enzootic areas of Asia and Africa (Carter \& de Alwis, 1989). The pathogen has also been associated with atrophic rhinitis and septicaemia of sheep (Krametter et al., 2004; Watson \& Davies, 2002). In addition, P. multocida is responsible for infections in deer (Aalbaek et al., 1999; Rimler et al., 1987), causes respiratory tract disease in rabbits (Lu et al., 1988; Rimler \& Brogden, 1986), and is associated with human infections resulting from cat and dog bites (Holm \& Tarnvik, 2000; Westling et al., 2000).

P. multocida strains express a polysaccharide capsule on 
their cell surfaces and the antigenic specificity of the capsule determines the organism's serogroup: A, B, D, E or F (Rimler \& Rhoades, 1989). It has long been recognized that strains of certain capsular serogroups are associated with specific diseases and animal species, which suggests that the capsular polysaccharide type plays a role in host and disease specificity. For example, the majority of cases of fowl cholera are caused by capsular type A strains (Rhoades \& Rimler, 1989), atrophic rhinitis of pigs is associated predominantly with capsular type D isolates (Chanter \& Rutter, 1989), bovine and porcine pneumonia are associated mainly with capsular type A strains (Chanter \& Rutter, 1989; Frank, 1989), and haemorrhagic septicaemia of cattle and water buffaloes is caused exclusively by capsular type B and E isolates (Carter \& de Alwis, 1989). However, the underlying genetic relationships of isolates representing different capsular types and associated with different host species and diseases is poorly understood.

P. multocida is classified into three subspecies based on DNA-DNA hybridization, namely $P$. multocida subsp. gallicida, P. multocida subsp. multocida and P. multocida subsp. septica (Mutters et al., 1985). The DNA-DNA hybridization study of Mutters et al. (1985) indicated that the three subspecies could be classified as distinct species, but this was considered pointless from the clinical viewpoint. These authors also demonstrated that fermentation of dulcitol and sorbitol could be used to differentiate between the three subspecies: P. multocida subsp. gallicida isolates fermented both dulcitol and sorbitol; P. multocida subsp. multocida isolates fermented sorbitol but not dulcitol; and P. multocida subsp. septica isolates fermented neither sugar alcohol. However, more recent data based on multilocus enzyme electrophoresis (MLEE) and ribotyping indicate that the three $P$. multocida subspecies do not represent distinct genotypic groups (Blackall et al., 1998; Muhairwa et al., 2001; Petersen et al., 1998, 2001). Furthermore, Kuhnert et al. (2000) showed that only two of six isolates that clustered as $P$. multocida subsp. septica by $16 \mathrm{~S}$ rRNA sequencing gave negative sorbitol reactions. Thus, the dulcitol and sorbitol fermentation patterns that define the three subspecies of $P$. multocida according to Mutters et al. (1985) do not accurately reflect the genetic relatedness of isolates. These conflicting results clearly indicate that the precise phylogenetic relationships of isolates representing each of these subspecies is complex and has yet to be satisfactorily resolved.

The genetic diversity of avian, bovine and porcine isolates of $P$. multocida have been investigated separately using a variety of molecular techniques, including restriction endonuclease analysis (Blackall et al., 2000; Rimler, 2000; Wilson et al., 1993), ribotyping (Blackall et al., 2000; Dabo et al., 1999; Petersen et al., 2001; Zhao et al., 1992), pulsed field gel electrophoresis (Gunawardana et al., 2000), repetitive sequence-based PCR and amplified fragment length polymorphism (Amonsin et al., 2002), and MLEE (Blackall et al., 1998). However, detailed comparative investigations of P. multocida isolates from poultry, cattle, sheep and pigs have not been undertaken and very little is known about the genetic relationships of such isolates. Strain diversity among a large collection of avian, bovine, ovine and porcine isolates of $P$. multocida has previously been examined by analysis of capsular polysaccharide and outer-membrane protein (OMP) variation (Davies et al., 2003a, b, c, 2004). Although these methods yield useful epidemiological data, they provide little information about the underlying genetic relatedness of isolates. The aim of the present study was to investigate the phylogenetic relationships of representative P. multocida isolates from these four host species by comparative sequence analysis of the 16S rRNA gene. Dulcitol and sorbitol fermentation patterns were also determined to establish correlations between these phenotypic characteristics and the phylogeny of $P$. multocida.

\section{METHODS}

Bacterial strains and growth conditions. Seventy-nine British field isolates of $P$. multocida were investigated in this study. These included 22 avian (poultry), 21 bovine, 10 ovine and 26 porcine isolates that were obtained from regional laboratories of the Veterinary Laboratories Agency (VLA) and originated from widespread geographic locations within England and Wales over a 13-year period (1987-99). The isolates were recovered mainly from diseased animals and were selected to represent the major variants associated with each host species as defined by their capsular polysaccharide and OMP types (Davies et al., 2003a, b, c, 2004). Two or more isolates were examined in those groups that represented the more common capsule/OMP type combinations. Four isolates originating from suspected cases of haemorrhagic septicaemia in cattle were also included in the study. Two of these (PM30 and PM36) were obtained from the National Collection of Type Cultures, whereas the other two (PM1192 and PM1200) originated from Pakistan and were provided by Dr R. Parton, University of Glasgow. In addition, the type strains of $P$. multocida subsp. gallicida (NCTC 10204), $P$. multocida subsp. multocida (NCTC 10322) and P. multocida subsp. septica (NCTC 11995) (obtained from the National Collection of Type Cultures) were included in the study. Properties of these isolates are presented in Table 1.

The isolates were stored at $-85^{\circ} \mathrm{C}$ in $50 \%(\mathrm{v} / \mathrm{v})$ glycerol in brain heart infusion broth (BHIB; Oxoid). Bacteria from $-85^{\circ} \mathrm{C}$ stock cultures were streaked onto blood agar [brain heart infusion agar containing $5 \%(\mathrm{v} / \mathrm{v})$ defibrinated sheep's blood] and incubated aerobically overnight at $37^{\circ} \mathrm{C}$. For preparation of DNA, a few colonies were inoculated into $15 \mathrm{ml}$ volumes of BHIB and grown overnight at $37^{\circ} \mathrm{C}$ with shaking at 120 r.p.m.

Dulcitol and sorbitol fermentation. Fermentation studies were performed using peptone water (Oxoid) containing $1 \%(\mathrm{w} / \mathrm{v})$ of either dulcitol or sorbitol and $18 \mathrm{mg}$ phenol red $\mathrm{l}^{-1}$ as indicator. A few colonies of overnight cultures were resuspended in $3 \mathrm{ml}$ volumes of PBS $(\mathrm{pH} \mathrm{7 \cdot 2)}$ and $50 \mu \mathrm{l}$ of this suspension was inoculated into $3 \mathrm{ml}$ volumes of dulcitol or sorbitol peptone water. The cultures were incubated at $37^{\circ} \mathrm{C}$ and the results recorded after 24,48 and $120 \mathrm{~h}$.

Preparation of chromosomal DNA. Cells from $1.0 \mathrm{ml}$ of overnight cultures were harvested by centrifugation for $1 \mathrm{~min}$ at $13000 \mathrm{~g}$ and washed once in sterile, distilled $\mathrm{H}_{2} \mathrm{O}$. DNA was prepared with the InstaGene Matrix (Bio-Rad) according to the manufacturer's instructions and stored at $-20^{\circ} \mathrm{C}$. 
Table 1. Properties of Pasteurella multocida isolates

Unless otherwise stated, all strains are P. multocida subsp. multocida. OMP types are unique for strains from each host species. AR, atrophic rhinitis; HS, haemorrhagic septicaemia; UT, untypable.

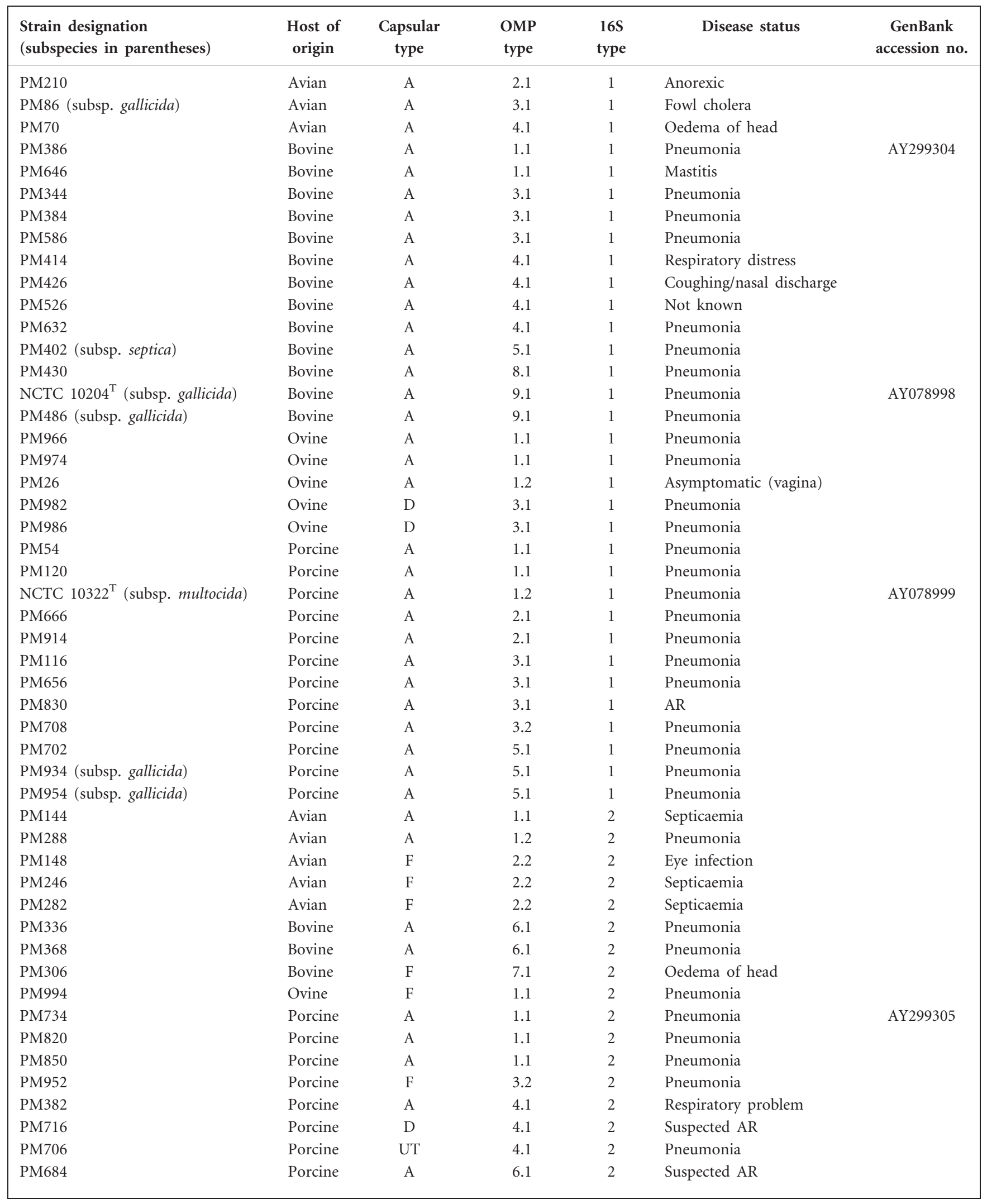


Table 1. cont.

\begin{tabular}{|c|c|c|c|c|c|c|}
\hline $\begin{array}{l}\text { Strain designation } \\
\text { (subspecies in parentheses) }\end{array}$ & $\begin{array}{l}\text { Host of } \\
\text { origin }\end{array}$ & $\begin{array}{l}\text { Capsular } \\
\text { type }\end{array}$ & $\begin{array}{l}\text { OMP } \\
\text { type }\end{array}$ & $\begin{array}{l}16 S \\
\text { type }\end{array}$ & Disease status & $\begin{array}{c}\text { GenBank } \\
\text { accession no. }\end{array}$ \\
\hline PM762 & Porcine & $\mathrm{D}$ & 6.1 & 2 & $\mathrm{AR}$ & \\
\hline PM230 & Avian & A & 9.1 & 3 & Septicaemia & \\
\hline PM316 & Bovine & A & 1.1 & 3 & Pneumonia & \\
\hline PM564 & Bovine & A & 2.1 & 3 & Pneumonia & AY299306 \\
\hline PM600 & Bovine & A & 2.1 & 3 & Pneumonia & \\
\hline PM628 & Bovine & A & 2.1 & 3 & Pneumonia & \\
\hline PM776 & Porcine & A & 3.2 & 3 & Pneumonia & \\
\hline PM44 & Ovine & $\mathrm{F}$ & 1.1 & 4 & Asymptomatic (vagina) & \\
\hline PM998 & Ovine & $\mathrm{F}$ & 1.1 & 4 & Pneumonia & \\
\hline PM2 & Ovine & $\mathrm{F}$ & 2.1 & 4 & Severe peritonitis & AY078996 \\
\hline PM8 & Ovine & $\mathrm{F}$ & 2.1 & 4 & Asymptomatic (vagina) & \\
\hline PM296 & Avian & $\mathrm{D}$ & 13.1 & 5 & Pneumonia & \\
\hline PM52 & Porcine & $\mathrm{D}$ & 1.2 & 5 & Pneumonia & \\
\hline PM758 & Porcine & $\mathrm{D}$ & 4.2 & 5 & $\mathrm{AR}$ & \\
\hline PM714 & Porcine & $\mathrm{D}$ & 6.1 & 5 & Pneumonia & AY299307 \\
\hline PM752 & Porcine & $\mathrm{D}$ & 6.1 & 5 & Pneumonia & \\
\hline PM818 & Porcine & $\mathrm{D}$ & 6.1 & 5 & Pneumonia & \\
\hline PM338 & Bovine & A & 4.1 & 6 & Pneumonia & AY299308 \\
\hline PM548 & Bovine & A & 5.3 & 7 & Vaginal discharge & AY299309 \\
\hline PM62 & Avian & B & 12.1 & 8 & Respiratory symptoms & AY299310 \\
\hline PM104 & Avian & A & 4.1 & 9 & Septicaemia & AY299311 \\
\hline PM36 (NCTC 10326) & Bovine & $\mathrm{E}$ & Not done & 10 & HS & AY324032 \\
\hline PM30 (NCTC 10323) & Bovine & $\mathrm{B}$ & Not done & 11 & HS & AY299312 \\
\hline PM1192 & Bovine & B & Not done & 11 & HS & \\
\hline PM1200 & Bovine & $\mathrm{B}$ & Not done & 11 & HS & \\
\hline PM74 & Avian & UT & 5.1 & 12 & Fowl cholera & AY299313 \\
\hline PM152 (subsp. septica) & Avian & A & 6.1 & 13 & Swollen joints & AY299314 \\
\hline PM214 & Avian & A & 8.1 & 13 & Septicaemia & \\
\hline NCTC $11995^{\mathrm{T}}$ (subsp. septica) & Feline & A & Unclassified & 14 & Abscess/cat bite & AY079000 \\
\hline PM64 & Avian & A & 11.1 & 15 & Fowl cholera & AY299315 \\
\hline PM106 (subsp. septica) & Avian & UT & 10.2 & 16 & Fowl cholera & AY299316 \\
\hline PM60 (subsp. septica) & Avian & UT & 10.1 & 17 & Septicaemia & AY299317 \\
\hline PM80 (subsp. septica) & Avian & A & 14.1 & 18 & Swollen heads & AY299318 \\
\hline PM82 & Avian & A & 7.1 & 19 & Swollen heads & AY299319 \\
\hline PM88 & Avian & A & 7.1 & 19 & High mortality & \\
\hline PM96 & Avian & A & 7.1 & 19 & Respiratory problems & \\
\hline
\end{tabular}

Amplification of the 16S rRNA gene and DNA sequence analysis. PCR fragments corresponding to nucleotides 20-1487 of the published $16 \mathrm{~S}$ rRNA gene sequence of $P$. multocida subsp. gallicida strain MCCM 00021 (GenBank accession no. AF224297) were amplified from the chromosomal DNA of all isolates with the universal primers $5^{\prime}$-AGAGTTTGATYMTGGC-3' (forward; positions 4-19) and 5'-GYTACCTTGTTACGACTT-3' (reverse; 15051488) (Davies et al., 1996). The $16 \mathrm{~S}$ rRNA gene fragments were amplified with a Taq DNA polymerase kit (Boehringer Mannheim) according to the manufacturer's instructions. PCR errors were shown to be negligible by amplifying and sequencing the $16 \mathrm{~S}$ rRNA gene twice in a small number of randomly selected isolates. PCRs were carried out in a Perkin Elmer 480 DNA thermal cycler using the following amplification parameters: denaturation at $94{ }^{\circ} \mathrm{C}$ for $45 \mathrm{~s}$, annealing at $50{ }^{\circ} \mathrm{C}$ for $45 \mathrm{~s}$ and extension at $72^{\circ} \mathrm{C}$ for $45 \mathrm{~s}$. Thirty cycles were performed and a final elongation step of $72^{\circ} \mathrm{C}$ for $10 \mathrm{~min}$ was used. Production of a PCR amplicon of the expected size was confirmed by agarose gel electrophoresis and the DNA purified with a QIAquick PCR purification kit (Qiagen). The DNA was finally eluted in $30 \mu \mathrm{l}$ sterile distilled $\mathrm{H}_{2} \mathrm{O}$ and stored at $-20^{\circ} \mathrm{C}$. Sequence reactions were performed with the ABI Prism Big Dye Terminator cycle sequencing kit (Applied Biosystems) in a GeneAmp PCR System 9700 (Applied Biosystems) thermal cycler and sequence analysis carried out with an Applied Biosystems 377 DNA Sequencer (University of Glasgow Sequencing Service). Both strands of the gene were sequenced in three overlapping segments using the following additional internal primers: 5'-AAGAAGCACCGGCTAACT-3' (forward/2; nucleotides 493-510), 5'-GCGAAGAACCTTACCTAC-3' (forward/3; nucleotides 967-984), 5'-GTAAGGTTCTTCGCGTTG-3' (reverse/2; nucleotides 980-963) and $5^{\prime}$ CCGGTGCTTCTTCTGTAA-3' (reverse/3; nucleotides 504-487). The primers were designed using the computer program Primer Designer (Version 2.0) and synthesized by Sigma-GenoSys.

Analysis of nucleotide sequence data. Nucleotide sequence data were analysed, edited and assembled with SEQED (Applied 
Biosystems) and the Lasergene (DNASTAR) sequence analysis software. Complete assembled sequences were aligned with CLUSTALX and statistical and phylogenetic analyses were conducted using MEGA version 2.1 (Kumar et al., 2001). The GenBank accession numbers for the 16S rRNA gene sequences corresponding to $16 \mathrm{~S}$ types 1-19 are provided in Table 1.

\section{RESULTS}

\section{Dulcitol and sorbitol fermentation}

Seventy-five isolates fermented sorbitol but not dulcitol and were classified as P. multocida subsp. multocida (Table 1). Five isolates fermented both dulcitol and sorbitol and were classified as $P$. multocida subsp. gallicida. These included isolates PM86 (avian), NCTC 10204 and PM486 (bovine), and PM934 and PM954 (porcine) (Table 1). The avian isolate represented capsule/OMP types $\mathrm{A} / 3.1$, the bovine isolates capsule/OMP types $\mathrm{A} / 9.1$ and the porcine isolates capsule/OMP types A/5.1 (Table 1). However, it has previously been shown that the avian OMP type 3.1, bovine OMP type 9.1 and porcine OMP type 5.1 profiles are very similar (they represent OMP group 4 - see Davies et al., 2004), suggesting that these isolates are closely related. Six isolates were unable to ferment either sugar alcohol and were classified as $P$. multocida subsp. septica. These included isolates PM60, PM80, PM106 and PM152 (avian), PM402 (bovine), and NCTC 11995 (feline) (Table 1). The avian isolates represented capsule/OMP types UT (untypable)/ 10.1 (PM60), A/14.1 (PM80), UT/10.2 (PM106) and A/6.1 (PM152), and the bovine isolate represented capsule/OMP types A/5.1 (Table 1).

\section{Nucleotide variation of the 16S rRNA gene}

A $1468 \mathrm{bp}$ fragment of the $16 \mathrm{~S}$ rRNA gene was sequenced in 86 isolates of $P$. multocida, including the type strains of the three subspecies, and 19 unique sequences, designated $16 \mathrm{~S}$ types 1-19, were identified (Fig. 1). There were 27 (1.8\%) polymorphic nucleotide sites among these sequences, pairwise differences ranged from 1 to $24(0.07$ to $1 \cdot 6 \%)$ nucleotides, and the mean number of nucleotide differences was $5 \cdot 38 \pm 1 \cdot 01$. However, visual comparison of the polymorphic nucleotide sites clearly indicates that the $16 \mathrm{~S}$ rRNA sequences consist of two distinct groups, A and B, represented by $16 \mathrm{~S}$ types $1-12$ and 13-19, respectively (Fig. 1). There were nine $(0.6 \%)$ polymorphic nucleotide sites among the group A sequences, pairwise differences ranged from 1 to $5(0.07$ to $0.34 \%)$ nucleotides, and the mean number of nucleotide differences was $1 \cdot 23 \pm 0 \cdot 60$. There were eight $(0 \cdot 54 \%)$ polymorphic nucleotide sites among the group B sequences, pairwise differences ranged from 1 to $7(0.07$ to $0.48 \%)$ nucleotides, and the mean number of nucleotide differences was $2 \cdot 84 \pm 1 \cdot 06$. In contrast, pairwise differences between group $\mathrm{A}$ and $\mathrm{B}$ sequences ranged from 18 to $24(1.23$ to $1.63 \%)$ nucleotides and the mean number of nucleotide differences was $21 \cdot 12 \pm 3 \cdot 90$. Pairwise differences among the avian sequences ranged from 1 to 23 nucleotides and the mean number of nucleotide
111111

11111244468999999999000124 967889344512778888999111153 813890803106784567689367413 ATGTAGGGAACTTAAGCTACTATACGG $\ldots \ldots \ldots \ldots \ldots \ldots$ $\ldots \ldots \ldots \ldots \ldots \ldots \ldots$ $\ldots \ldots \ldots$. . . . . . . . $\ldots \ldots \ldots \ldots \ldots \ldots \ldots$ $\ldots \ldots \ldots \ldots \ldots \ldots \ldots \ldots$

$\mathrm{G} \ldots \ldots \ldots \ldots \ldots \ldots \ldots$ $\ldots \ldots \ldots \ldots \ldots \ldots \ldots$ $\ldots \ldots \ldots . . \ldots \ldots \ldots \ldots$ $\ldots \ldots \ldots \ldots \ldots \ldots \ldots \ldots \ldots$ $\ldots \ldots \ldots \ldots$. . . . . . . . . . АT ............... .ATCTT... . GT . AGCTTGCAGGCTA. A . ATCTT . . GT . AGCTTGCAGGCTA. . . ATCTT . . . GT . AGCTTGCAGGCT . . A . A.ATTAATGT . AGCTTGCAGGCT . . A .A.ATT . ATGT . AGCTTGCAGGCT . . A . ATATT . . TG . AGCTTGCAGGCTA.A .ATATT . . TGT . AGCTTGCAGGCTA. A

Fig. 1. Distribution of polymorphic nucleotide sites among the 19 16S rRNA alleles of $P$. multocida. 16S types are shown at the left of each sequence. The vertical numbers above the sequences represent the positions of polymorphic nucleotide sites within the $1468 \mathrm{bp}$ segment. The dots represent sites where the nucleotides match those of the first (topmost) sequence.

differences was $11 \cdot 75 \pm 2 \cdot 19$. In contrast, pairwise differences among the bovine, ovine and porcine sequences ranged from 1 to 3,1 to 2 , and 1 to 3 nucleotides, respectively, and the mean number of nucleotide differences was $1.12 \pm 0.52,1.09 \pm 0.84$ and $0.91 \pm 0.59$, respectively. Fifty-nine per cent of the isolates were associated with $16 \mathrm{~S}$ types 1 (33 isolates) or 2 (18), $19 \%$ of the isolates contained $16 \mathrm{~S}$ types 3 (6), 4 (4) or 5 (6), and $22 \%$ of the isolates possessed 16S types 6-19 (19) (Table 1).

\section{Phylogenetic relationships of $\boldsymbol{P}$. multocida isolates in relation to the host of origin and capsular polysaccharide and OMP types}

A neighbour-joining dendrogram representing the phylogenetic relationships of the $8616 \mathrm{~S}$ rRNA gene sequences is shown in Fig. 2. The host of origin, capsular polysaccharide types and OMP types of each isolate are also shown. The $16 \mathrm{~S}$ rRNA sequences were represented by two major lineages, A and $\mathrm{B}$, which correspond to the two groups of $16 \mathrm{~S}$ types described above. The branching of lineages $\mathrm{A}$ and $\mathrm{B}$ was extremely robust, as indicated by the high bootstrap value of $99 \%$. In contrast, the bootstrap values for many of the finer branches were relatively small $(<50 \%)$ because of the high similarity of the sequences. Lineage A (76 isolates) was represented by isolates from all four host species, whereas lineage B (10 isolates) was represented exclusively by isolates of avian origin as well as the feline P. multocida subsp. septica type strain. Lineage A itself consisted of three 


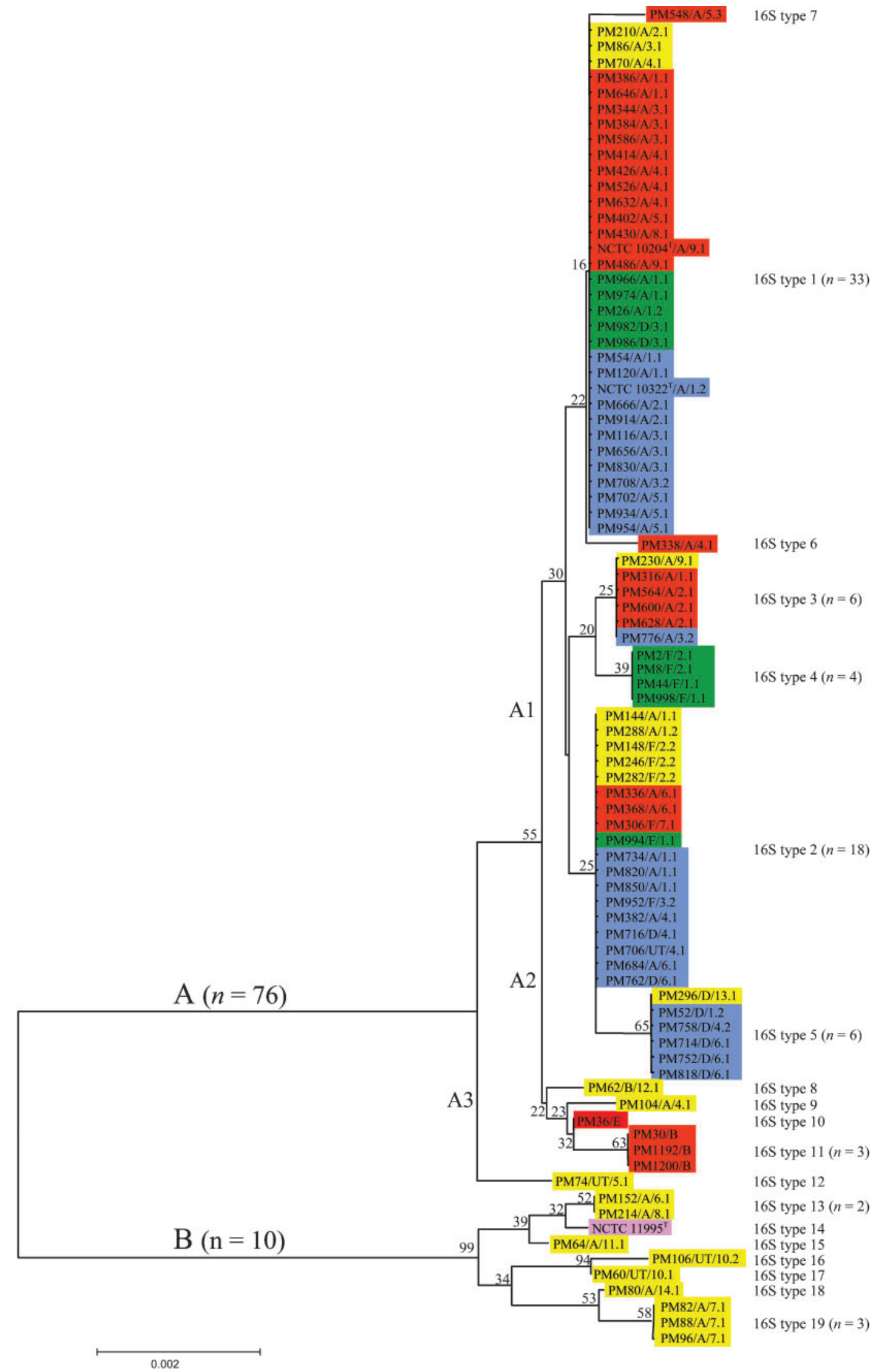


clusters, A1-A3. Cluster A1 comprised 69 (91\%) of the lineage $\mathrm{A}$ isolates and included isolates from all four host species. Cluster A2 was represented by six isolates which included the four haemorrhagic septicaemia isolates as well as two avian isolates. Notably, three of the haemorrhagic septicaemia isolates and one of the avian isolates were of capsular type B; the fourth haemorrhagic septicaemia isolate was of capsular type $\mathrm{E}$ and the second avian isolate was of capsular type A. Cluster A3 included a single untypable avian isolate. In lineage A, $16 \mathrm{~S}$ types 1 and 2 were associated with isolates from all four host species, 16S type 3 was present in avian, bovine and porcine isolates, and 16S type 5 occurred in avian and porcine isolates. In contrast, $16 \mathrm{~S}$ type 4 was associated exclusively with ovine isolates, $16 \mathrm{~S}$ types $6,7,10$ and 11 with bovine isolates and 16S types 8,9 and 12 with avian isolates. In lineage B, $16 \mathrm{~S}$ types 13 , $15,16,17,18$ and 19 were associated with avian isolates, whereas $16 \mathrm{~S}$ type 14 was associated with the feline P. multocida subsp. septica type strain.

Avian isolates. The $16 \mathrm{~S}$ rRNA sequences of the avian isolates were extremely diverse in comparison to those of the bovine, ovine and porcine isolates (Fig. 2). As well as being the only host species represented in lineage B (with the exception of the feline subsp. septica type strain), avian isolates also possessed the most divergent sequences within lineage A. Avian isolates were associated with seven $16 \mathrm{~S}$ types in lineage A and six in lineage B. Whereas $16 \mathrm{~S}$ types $1-3$ and 5 (lineage A) were present in isolates from other host species, the remaining nine $16 \mathrm{~S}$ types were unique to avian isolates. 16S types $8,9,12$ and 15-19 were associated with capsule/OMP types $\mathrm{B} / 12.1$ (one isolate), A/4.1 (1), UT/5.1 (1), A/11.1 (1), UT/10.2 (1), UT/ 10.1 (1), A/14.1 (1) and A/7.1 (3), respectively. In contrast, avian isolates of $16 \mathrm{~S}$ types 1,2 and 13 were associated with capsule/OMP types $\mathrm{A} / 2.1$ (1), A/3.1 (1) and $\mathrm{A} / 4.1$ (1), A/1.1 (1), A/1.2 (1) and F/2.2 (3), and A/6.1 (1) and A/8.1 (1), respectively.

Bovine isolates. Bovine isolates were represented primarily by $16 \mathrm{~S}$ types 1 (13 isolates), 2 (3), 3 (4) and 11 (3), although an additional three isolates were associated with $16 \mathrm{~S}$ types 6,7 and 10 (Fig. 2). 16S type 1 was associated with capsular type A isolates of OMP types 1.1 (two isolates), 3.1 (3), 4.1 (4), 5.1 (1), 8.1 (1) and 9.1 (2), whereas $16 \mathrm{~S}$ type 2 was present in isolates of capsule/ OMP types A/6.1 (2) and F/7.1 (1), and 16S type 3 occurred in capsular type A isolates of OMP types 1.1 (1) and $2.1(3) .16 \mathrm{~S}$ types 10 and 11 were associated with capsular type E (1) and B (3) isolates, respectively, which were isolated from cases of haemorrhagic septicaemia (the OMP types of these isolates were not determined).
Ovine isolates. Ovine isolates were represented primarily by $16 \mathrm{~S}$ types 1 (five isolates) and 4 (4); a single isolate was associated with $16 \mathrm{~S}$ type 2 (Fig. 2). $16 \mathrm{~S}$ type 1 was associated with capsular type A isolates of OMP types 1.1 (2) and 1.2 (1) and capsular type D isolates of OMP type 3.1 (2), whereas $16 \mathrm{~S}$ type 4 was present in capsular type $\mathrm{F}$ isolates of OMP types 1.1 (2) and 2.1 (2). The single $16 \mathrm{~S}$ type 2 isolate was of capsular type F and OMP type 1.1.

Porcine isolates. Porcine isolates were represented by $16 \mathrm{~S}$ types 1 (12 isolates), 2 (9) and 5 (5); a single isolate possessed 16S type 3 (Fig. 2). 16S type 1 was associated with capsular type A isolates of OMP types 1.1 (2), 1.2 (1), 2.1 (2), 3.1 (3), 3.2 (1) and 5.1 (3), whereas 16S type 2 was present in capsular type A isolates of OMP types 1.1 (3), 4.1 (1), 6.1 (1), capsular type D isolates of OMP types 4.1 (1) and 6.1 (1), a capsular type F isolate of OMP type 3.2 and an untypable isolate of OMP type 4.1, and $16 \mathrm{~S}$ type 5 occurred in capsular type $\mathrm{D}$ isolates of OMP types $1.2(1), 4.2(1)$ and $6.1(3)$.

\section{Phylogenetic relationships of $\boldsymbol{P}$. multocida isolates representing 19165 types in relation to other Pasteurella multocida strains and Pasteurella species}

The phylogenetic relationships of the 19 P. multocida $16 \mathrm{~S}$ rRNA sequences obtained in the present study were compared to those from biovar 2 Pasteurella avium and Pasteurella canis isolates that have recently been reclassified as P. multocida (Christensen et al., 2004) (Fig. 3). The sequences were also compared with those from three feline P. multocida subsp. septica isolates (Kuhnert et al., 2000) and from the type strains of the related species Pasteurella dagmatis, P. canis, Pasteurella stomatis, P. avium, Pasteurella gallinarum and Pasteurella volantium (Korczak et al., 2004) (Fig. 3). The sequences were aligned and compared over the same $1468 \mathrm{bp}$ segment corresponding to $16 \mathrm{~S}$ types $1-19$. The dendrogram was constructed using the pairwise deletion option to handle missing data because the sequences were of unequal lengths. The biovar $2 P$. avium and $P$. canis sequences (Christensen et al., 2004) were identical and together formed a lineage that was most closely related to the cluster of isolates in lineage A represented by PM386 (16S type 1), PM548 (16S type 7) and PM338 (16S type 6) (Fig. 3). The three feline $P$. multocida subsp. septica sequences (Kuhnert et al., 2000) were associated with lineage $B$ and were most closely related to the $16 \mathrm{~S}$ type 13 sequence of avian isolate PM152 (Fig. 3). The 16S rRNA sequences of the type strains of $P$. dagmatis, $P$. canis and $P$. stomatis, and P. avium, $P$. gallinarum and $P$. volantium, represented two distinct

Fig. 2. Neighbour-joining dendrogram representing the phylogenetic relationships of the $16 \mathrm{~S}$ rRNA gene of 86 P. multocida isolates. Avian, bovine, ovine, porcine and feline isolates are highlighted in yellow, red, green, blue and pink, respectively. The strain numbers, capsular types and OMP types are provided for each isolate. The OMP types are unique to strains from each host species. Bootstrap values are shown adjacent to each node. 


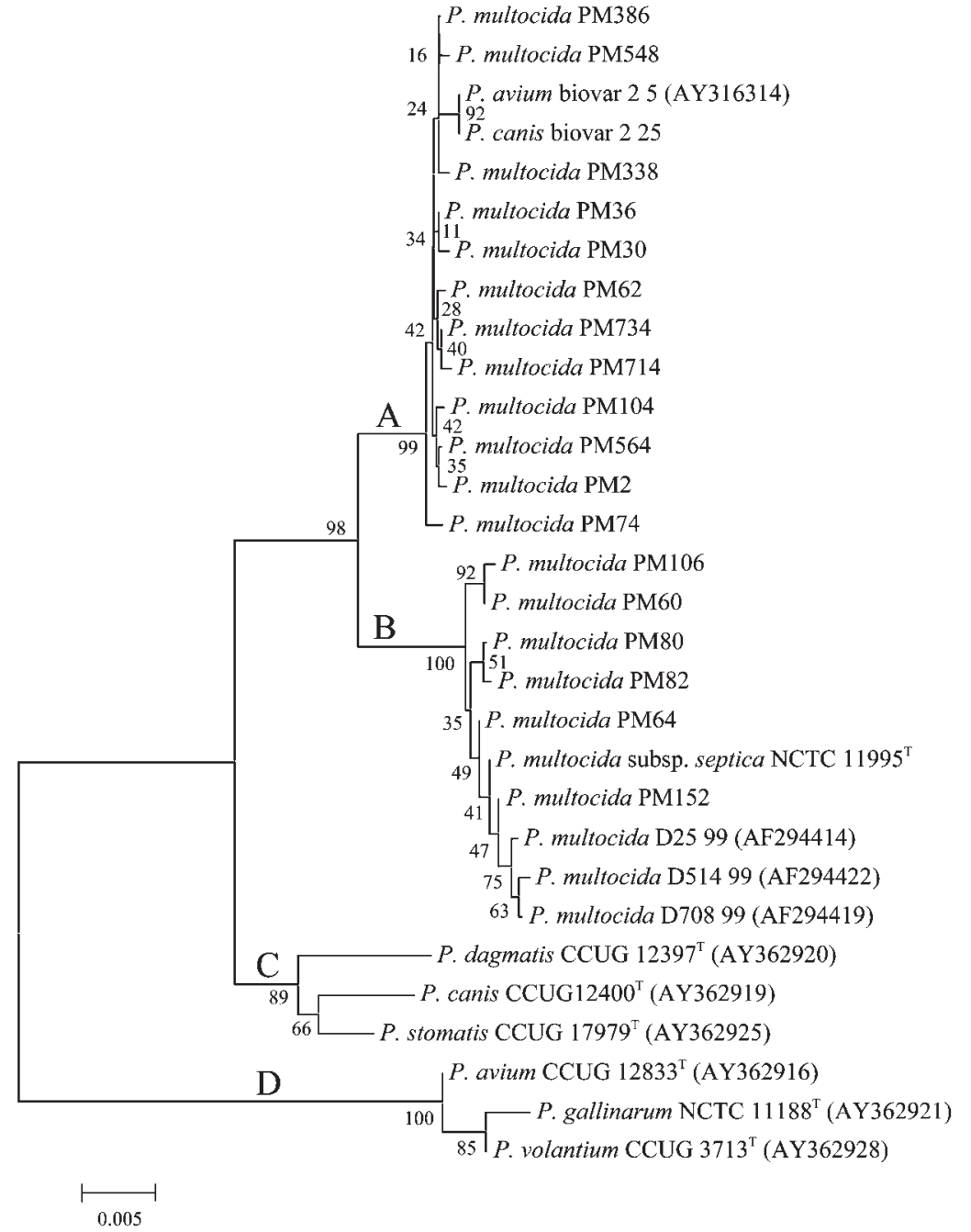

Fig. 3. Neighbour-joining dendrogram representing the phylogenetic relationships of the 16S rRNA gene of $P$. multocida strains of 16S types 1-19 in relation to the $16 \mathrm{~S}$ rRNA gene of biovar $2 P$. avium and $P$. canis isolates (Christensen et al., 2004), feline $P$. multocida subsp. septica isolates (D25 99, D514 99, D708 99) (Kuhnert et al., 2000), and other Pasteurella species (Korczak et al., 2004). The dendrogram was constructed using the pairwise deletion option to handle missing data. Bootstrap values are shown adjacent to each node. clusters, C and D, respectively. There were 28 (1.91\%) polymorphic nucleotide sites among the three sequences of lineage $\mathrm{C}$, pairwise differences ranged from 14 to 25 $(0.95$ to $1.70 \%)$, and the mean number of polymorphic nucleotide sites was $18 \cdot 67 \pm 3 \cdot 39$. In contrast, there were only $2(0 \cdot 14 \%)$ polymorphic nucleotide sites among the three sequences of lineage $\mathrm{D}$, pairwise differences ranged from 1 to $2(0 \cdot 07$ to $0 \cdot 14 \%)$, and the mean number of polymorphic nucleotide sites was $1 \cdot 33 \pm 0 \cdot 96$.

\section{DISCUSSION}

Previous investigations of genetic diversity and relationships among $P$. multocida isolates have usually focused on isolates originating from a single host species (Amonsin et al., 2002; Blackall et al., 1998, 2000; Dabo et al., 1999; Gardner et al., 1994; Gunawardana et al., 2000; Petersen et al., 2001; Rimler, 2000; Wilson et al., 1993; Zhao et al., 1992). Furthermore, wider taxonomic studies of the Pasteurellaceae have included only small numbers of $P$. multocida isolates (usually the type strains), which are not necessarily representative of natural populations of the species (Dewhirst et al., 1992; Korczak et al., 2004; Mutters et al., 1985; Olsen et al., 2003). In the present study, a 1468 bp segment of the 16S rRNA gene was sequenced in 86 isolates of $P$. multocida, including the type strains of the three subspecies. Seventy-nine of these isolates were obtained from wide geographic regions of England and Wales and were selected to represent the majority of groups previously defined by variation of capsular polysaccharide and OMP types among avian, bovine, ovine and porcine isolates (Davies et al., 2003a, b, c, 2004). The present study effectively represents a comparative analysis of over 446 $P$. multocida isolates from the four major domesticated species within England and Wales.

It has previously been suggested that patterns of dulcitol and sorbitol fermentation are indicators of the three P. multocida subspecies (Mutters et al., 1985). However, a number of studies, mostly involving avian isolates but using a variety of different techniques, suggest that the three subspecies, particularly P. multocida subsp. multocida and P. multocida subsp. septica, do not represent distinct genotypic groups (Blackall et al., 1998; Muhairwa et al., 2001; Petersen et al., 1998, 2001). In particular, Kuhnert 
et al. (2000) demonstrated that only two of six isolates classified as P. multocida subsp. septica by $16 \mathrm{~S}$ rRNA sequence determination gave a negative reaction for sorbitol fermentation. In the present study, $87 \%$ of the isolates were classified as $P$. multocida subsp. multocida by dulcitol and sorbitol fermentation patterns, but these isolates have diverse $16 \mathrm{~S}$ rRNA gene sequences that were represented in both lineages A and B (Table 1 and Fig. 2). These isolates also have very diverse OMP profiles and were associated with all four of the host species. Avian P. multocida subsp. septica isolates were associated exclusively with lineage B, and this finding is in agreement with that of Blackall et al. (1998) who placed all of their avian P. multocida subsp. septica isolates into a single group (ribotype cluster R1/ MLEE cluster B). However, the presence of bovine $P$. multocida subsp. septica isolates within lineage A indicates that this phenotype is not restricted to avian isolates of lineage B. Avian P. multocida subsp. multocida isolates were also associated with lineage $B$, although these could be distinguished from the P. multocida subsp. septica isolates of the same lineage by differences in their capsule/ OMP and $16 \mathrm{~S}$ types. However, a wide range of genetically diverse avian $P$. multocida subsp. multocida isolates was also associated with lineage A. These observations clearly support the view that dulcitol and sorbitol fermentation are not accurate indicators of genetic relatedness among P. multocida isolates (Blackall et al., 1998; Kuhnert et al., 2000; Muhairwa et al., 2001; Petersen et al., 1998, 2001). In general, the existence of phenotypic variation in key characters of P. multocida (Christensen et al., 2004) does not provide a logical basis for the use of phenotypic characterization in the classification of this species.

The possession of similar OMP profiles (Davies et al., 2004) and identical 16 S types (Table 1) by all the isolates classified as P. multocida subsp. gallicida (recovered from birds, cattle and pigs) provides strong evidence that they represent a more homogeneous and closely related group than isolates classified as $P$. multocida subsp. multocida and P. multocida subsp. septica. Ribotyping studies have similarly shown that both avian (Blackall et al., 1998; Petersen et al., 2001) and porcine (Bowles et al., 2000) isolates of $P$. multocida subsp. gallicida represent welldefined groups that are genetically distinct from other isolates. Together, the OMP, $16 \mathrm{~S}$ rRNA and fermentation data suggest that $P$. multocida subsp. gallicida isolates represent a distinct clone or clone complex within $P$. multocida. The possession of identical $16 \mathrm{~S}$ types (i.e. $16 \mathrm{~S}$ type 1 ) by isolates phenotypically classified as P. multocida subsp. gallicida and P. multocida subsp. multocida (Table 1) does not support the view that they represent distinct species, or even subspecies (Mutters et al., 1985), and raises questions about the taxonomic status of those isolates that have more divergent $16 \mathrm{~S}$ rRNA genes. In particular, serotype $\mathrm{B}$ and $\mathrm{E}$ isolates associated with haemorrhagic septicaemia of cattle and water buffaloes possess $16 \mathrm{~S}$ types 11 and 10 (cluster A2), respectively, and are clearly more divergent from $P$. multocida subsp. multocida and
P. multocida subsp. gallicida isolates of $16 \mathrm{~S}$ type 1 (cluster A1) than the latter are to each other (Fig. 2).

The 16S rRNA sequence data confirmed the conclusions of previous studies that avian P. multocida isolates are extremely diverse (Blackall et al., 1995, 1998; Carpenter et al., 1991; Christiansen et al., 1992; Davies et al., 2003b; Gunawardana et al., 2000; Petersen et al., 2001; Snipes et al., 1989; Wilson et al., 1993, 1995). The data also show that avian isolates are substantially more diverse than bovine, ovine and porcine isolates (Fig. 2). The avian isolates were associated with $1316 \mathrm{~S}$ types in lineages A (seven) and B (six), whereas the bovine, ovine and porcine isolates were associated with seven, three, and four $16 \mathrm{~S}$ types, respectively, in lineage A. The mean number of nucleotide differences was also substantially higher for the avian $(11 \cdot 75 \pm 2 \cdot 19)$ isolates than for the bovine $(1 \cdot 12 \pm 0 \cdot 52)$, ovine $(1 \cdot 09 \pm 0.84)$, and porcine $(0.91 \pm 0.59)$ isolates. Possible reasons for the greater diversity of the avian strains have been discussed previously (Davies et al., 2003b). There were also marked differences in the association of $16 \mathrm{~S}$ types with the hosts of origin between strains of lineages A and B. The $16 \mathrm{~S}$ types of lineage A were generally associated with a wide range of host species but, with a single exception, all of the $16 \mathrm{~S}$ types of lineage B were recovered from birds. These observations suggest that avian, bovine, ovine and porcine isolates of lineage A have evolved from a common ancestor and have become adapted to different host species, whereas avian strains of lineage B have diverged from a different common avian ancestor and have remained highly adapted to birds.

There were strong correlations between 16S types and capsule/OMP types among isolates from each of the four host species, which suggests that isolates of the same capsule/ OMP types are genetically related and represent distinct clones. In the case of the bovine isolates, the relationship between OMP type and 16S type shows partial agreement with data based on the concatenated sequences of seven housekeeping enzyme genes (Davies et al., 2004). Isolates of OMP types 1.1 and 2.1, 3.1, 4.1 and 8.1, and 6.1 represent three distinct clusters for both housekeeping enzyme and $16 \mathrm{~S}$ rRNA genes, but the housekeeping genes of isolates of OMP types 5.1, 5.3, 7.1 and 9.1 are significantly more divergent than the corresponding $16 \mathrm{~S}$ rRNA genes (Davies et al., 2004). The incongruent topologies for the $16 \mathrm{~S}$ rRNA and concatenated housekeeping enzyme gene trees suggest that recombination has influenced the apparent phylogenies of these genes. Further evidence for recombination is provided by the association of the same OMP types with different $16 \mathrm{~S}$ types for a given host. Recombination of the 16S rRNA gene has also been described in Neisseria meningitidis (Sacchi et al., 2002) and Streptococcus anginosus (Schouls et al., 2003). Sequence analysis of the slowly evolving $16 \mathrm{~S}$ rRNA gene is incapable of detecting the finer genetic variation that exists between isolates from the same host species and which has previously been demonstrated by analysis of OMP profiles (Davies et al., 2003a, b, c, 2004). 
The presence of very different OMP profiles in isolates of the same $16 \mathrm{~S}$ types indicates that cell-surface proteins are evolving more rapidly than the $16 \mathrm{~S}$ rRNA gene. In particular, the presence of very different OMP profiles in strains of the same, or similar, 16S types, but from different host species, suggests a correlation between OMP diversification and host adaptation.

The capsular type B and E isolates associated with haemorrhagic septicaemia of cattle and water buffaloes have very similar 16S rRNA sequences (16S types 11 and 10, respectively) and are closely related to two avian capsular type A and B isolates (16S types 9 and 8, respectively). These isolates represent a distinct branch within lineage A (cluster A2) and have clearly diverged from those P. multocida isolates (cluster A1) that are associated with avian fowl cholera, pneumonia of cattle, sheep and pigs, and atrophic rhinitis of pigs (Table 1 and Fig. 2). These data represent the first $16 \mathrm{~S}$ rRNA sequence comparison of capsular type B and $\mathrm{E}$ isolates and clearly demonstrate their phylogenetic position within the species $P$. multocida. The $16 \mathrm{~S}$ type 8 isolate PM62 represents one of only four capsular type B (OMP type 12.1) isolates that were recovered from poultry (Davies et al., 2003b). Capsular type B P. multocida isolates are generally recovered infrequently from birds (Rhoades \& Rimler, 1987; Wilson et al., 1993). The findings indicate that capsular type B isolates associated with haemorrhagic septicaemia of cattle and water buffaloes and those recovered from birds have common evolutionary origins. Capsular type $\mathrm{F}$ is also infrequently recovered from birds and other species (Rhoades \& Rimler, 1987; Wilson et al., 1993). However, with the exception of four ovine capsular type $\mathrm{F}$ isolates of $16 \mathrm{~S}$ type 4 , it was notable that all of the capsular type F strains examined in this study, which were isolated from all four host species, possessed $16 \mathrm{~S}$ type 2 (Fig. 2). These findings suggest that capsular type $\mathrm{F}$ isolates associated with different host species also have common evolutionary origins.

Biovar 2 strains of $P$. avium and P. canis (formerly classified as Bisgaard Taxon 13) both gave positive reactions in a P. multocida-specific 23S rRNA-based PCR assay (Miflin \& Blackall, 2001) and were recently reclassified as $P$. multocida by DNA-DNA hybridization and $16 \mathrm{~S}$ rRNA sequence comparison (Christensen et al., 2004). In the present study, the biovar $2 P$. avium and $P$. canis strains were shown to be closely related to $P$. multocida isolates represented by $16 \mathrm{~S}$ types 1 (PM386), 6 (PM338) and 7 (PM548) of lineage A (Fig. 3). Interestingly, isolates of $16 \mathrm{~S}$ types 1, 6 and 7 are all associated with the bovine host, and this is also the case for biovar 2 strains of $P$. avium and $P$. canis (Christensen et al., 2004). These findings not only provide support for the 23S rRNA-based PCR identification of P. multocida (Miflin \& Blackall, 2001), but also provide clarification of the phylogenetic position of these strains within the species. Three 16S rRNA sequences representing feline $P$. multocida subsp. septica isolates (Kuhnert et al., 2000) were shown to be closely related to avian $P$. multocida isolates and to the feline type strain of P. multocida subsp. septica of lineage B (Fig. 3). The emerging picture is that isolates representing lineage $\mathrm{B}$ of P. multocida are associated almost exclusively with birds and cats. Presumably, cats have acquired avian-adapted strains of $P$. multocida as a consequence of their predatorprey relationship.

The current subspecies nomenclature of $P$. multocida neither accurately reflects the $16 \mathrm{~S}$ rRNA-based phylogenetic relationships among isolates nor does it adequately encompass the full range of diversity within the species. Kuhnert et al. (2000) proposed that 16S rRNA sequences, rather than sorbitol fermentation, should be used for the accurate identification of $P$. multocida subsp. septica isolates and their unambiguous differentiation from $P$. multocida subsp. multocida and P. multocida subsp. gallicida. Based on their relatively high 16S rRNA sequence divergence, these authors also suggested that isolates classified as P. multocida subsp. septica might be considered a separate species. The mean number of nucleotide differences $(21 \cdot 12 \pm 3 \cdot 90)$ between the sequences of lineages A and B ( $P$. multocida sequences) was marginally greater than the value $(18 \cdot 67 \pm 3 \cdot 39)$ for lineage $C$ ( $P$. dagmatis, $P$. canis and $P$. stomatis sequences) but was substantially higher than the value $(1.33 \pm 0.96)$ for lineage $\mathrm{D}(P$. avium, $P$. gallinarum and $P$. volantium sequences) (Fig. 3). Together with the DNA-DNA hybridization data of Mutters et al. (1985) and the pronounced differences in host association of strains representing lineages A (birds, cattle, pigs and sheep) and B (birds and cats), these data provide strong support for the suggestion that lineage B ( $P$. multocida subsp. septica) be reclassified as a separate species (Kuhnert et al., 2000).

In conclusion, this study provides a $16 \mathrm{~S}$ rRNA-based evolutionary framework for $P$. multocida isolates recovered from birds, cattle, pigs and sheep in England and Wales. Dulcitol and sorbitol fermentation patterns do not correlate with genetic relatedness, and the current subspecies nomenclature neither accurately reflects the 16S rRNAbased phylogenetic relationships among isolates nor adequately encompasses the full range of diversity within the species. P. multocida consists of two divergent lineages, $A$ and $B$, and evidence is presented to support the suggestion that lineage B (P. multocida subsp. septica) might represent a separate species.

\section{ACKNOWLEDGEMENTS}

This study was supported by a Wellcome Trust University Award (053669/Z/98/Z). Staff of the Veterinary Laboratories Agency (VLA) are gratefully acknowledged for the provision of isolates, particularly those at Bury St Edmunds, for making available strains of their culture collection.

\section{REFERENCES}

Aalbaek, B., Eriksen, L., Rimler, R. B., Leifsson, P. S., Basse, A., Christiansen, T. \& Eriksen, E. (1999). Typing of Pasteurella 
multocida from haemorrhagic septicaemia in Danish fallow deer (Dama dama). APMIS 107, 913-920.

Amonsin, A., Wellehan, J. F. X., Li, L. L., Laber, J. \& Kapur, V. (2002). DNA fingerprinting of Pasteurella multocida recovered from avian sources. J Clin Microbiol 40, 3025-3031.

Blackall, P. J., Pahoff, J. L., Marks, D., Fegan, N. \& Morrow, C. J. (1995). Characterisation of Pasteurella multocida isolated from fowl cholera outbreaks on turkey farms. Aust Vet J 72, 135-138.

Blackall, P. J., Fegan, N., Chew, G. T. I. \& Hampson, D. J. (1998). Population structure and diversity of avian isolates of Pasteurella multocida from Australia. Microbiology 144, 279-289.

Blackall, P. J., Fegan, N., Pahoff, J. L. \& 8 other authors (2000). The molecular epidemiology of four outbreaks of porcine pasteurellosis. Vet Microbiol 72, 111-120.

Bowles, R. E., Pahoff, J. L., Smith, B. N. \& Blackall, P. J. (2000). Ribotype diversity of porcine Pasteurella multocida from Australia. Aust Vet J 78, 630-635.

Carpenter, T. E., Snipes, K. P., Kasten, R. W., Hird, D. W. \& Hirsh, D. C. (1991). Molecular epidemiology of Pasteurella multocida in turkeys. Am J Vet Res 52, 1345-1349.

Carter, G. R. \& de Alwis, M. C. L. (1989). Haemorrhagic septicaemia. In Pasteurella and Pasteurellosis, pp. 131-160. Edited by C. F. Adlam \& J. M. Rutter. London: Academic Press.

Chanter, N. \& Rutter, J. M. (1989). Pasteurellosis in pigs and the determinants of virulence of toxigenic Pasteurella multocida. In Pasteurella and Pasteurellosis, pp. 161-195. Edited by C. F. Adlam \& J. M. Rutter. London: Academic Press.

Christensen, H., Angen, O., Olsen, J. E. \& Bisgaard, M. (2004). Revised description and classification of atypical isolates of Pasteurella multocida from bovine lungs based on genotypic characterization to include variants previously classified as biovar 2 of Pasteurella canis and Pasteurella avium. Microbiology 150, 1757-1767.

Christiansen, K. H., Carpenter, T. E., Snipes, K. P. \& Hird, D. W. (1992). Transmission of Pasteurella multocida on California turkey premises in 1988-89. Avian Dis 36, 262-271.

Dabo, S. M., Debey, B. M., Montelongo, M. \& Confer, A. W. (1999). Genomic DNA restriction site heterogeneity in bovine Pasteurella multocida serogroup A isolates detected with an rRNA probe. J Med Microbiol 48, 279-286.

Davies, R. L., Paster, B. J. \& Dewhirst, F. E. (1996). Phylogenetic relationships and diversity within the Pasteurella haemolytica complex based on 16S rRNA sequence comparison and outer membrane protein and lipopolysaccharide analysis. Int J Syst Bacteriol 46, 736-744.

Davies, R. L., MacCorquodale, R., Baillie, S. \& Caffrey, B. (2003a). Characterization and comparison of Pasteurella multocida strains associated with porcine pneumonia and atrophic rhinitis. J Med Microbiol 52, 59-67.

Davies, R. L., MacCorquodale, R. \& Caffrey, B. (2003b). Diversity of avian Pasteurella multocida strains based on capsular PCR typing and variation of the OmpA and $\mathrm{OmpH}$ outer membrane proteins. Vet Microbiol 91, 169-182.

Davies, R. L., Watson, P. J. \& Caffrey, B. (2003c). Comparative analyses of Pasteurella multocida strains associated with the ovine respiratory and vaginal tracts. Vet $\operatorname{Rec} 152,7-10$.

Davies, R. L., MacCorquodale, R. \& Reilly, S. (2004). Characterisation of bovine strains of Pasteurella multocida and comparison with isolates of avian, ovine and porcine origin. Vet Microbiol 99, 145-158.

Dewhirst, F. E., Paster, B. J., Olsen, I. \& Fraser, G. J. (1992) Phylogeny of 54 representative strains of species in the family Pasteurellaceae as determined by comparison of $16 \mathrm{~S}$ rRNA sequences. J Bacteriol 174, 2002-2013.
Frank, G. H. (1989). Pasteurellosis of cattle. In Pasteurella and Pasteurellosis, pp. 197-222. Edited by C. F. Adlam \& J. M. Rutter. London: Academic Press.

Gardner, I. A., Kasten, R., Eamens, G. J., Snipes, K. P. \& Anderson, R. J. (1994). Molecular fingerprinting of Pasteurella multocida associated with progressive atrophic rhinitis in swine herds. $J$ Vet Diagn Invest 6, 442-447.

Gunawardana, G. A., Townsend, K. M. \& Frost, A. J. (2000). Molecular characterisation of avian Pasteurella multocida isolates from Australia and Vietnam by REP-PCR and PFGE. Vet Microbiol 72, 97-109.

Holm, M. \& Tarnvik, A. (2000). Hospitalization due to Pasteurella multocida-infected animal bite wounds: correlation with inadequate primary antibiotic medication. Scand J Infect Dis 32, 181-183.

Korczak, B., Christensen, H., Emler, S., Frey, J. \& Kuhnert, P. (2004). Phylogeny of the family Pasteurellaceae based on rpoB sequences. Int J Syst Evol Microbiol 54, 1393-1399.

Krametter, R., Hassan, J., Mayrhofer, E. \& Baumgartner, W. (2004). Atrophic rhinitis in sheep in Austria. Vet Rec 154, 147-148.

Kuhnert, P., Boerlin, P., Emler, S., Krawinkler, M. \& Frey, J. (2000). Phylogenetic analysis of Pasteurella multocida subspecies and molecular identification of feline P. multocida subsp. septica by $16 \mathrm{~S}$ rRNA gene sequencing. Int J Med Microbiol 290, 599-604.

Kumar, S., Tamura, K., Jakobsen, I. B. \& Nei, M. (2001). MEGA2: molecular evolutionary genetics analysis software. Bioinformatics $\mathbf{1 7}$, 1244-1245.

Lu, Y. S., Afendis, S. J. \& Pakes, S. P. (1988). Identification of immunogenic outer membrane proteins of Pasteurella multocida 3 : A in rabbits. Infect Immun 56, 1532-1537.

Miflin, J. K. \& Blackall, P. J. (2001). Development of a 23 S rRNAbased PCR assay for the identification of Pasteurella multocida. Lett Appl Microbiol 33, 216-221.

Muhairwa, A. P., Christensen, J. P. \& Bisgaard, M. (2001). Relationships among Pasteurellaceae isolated from free ranging chickens and their animal contacts as determined by quantitative phenotyping, ribotyping and REA-typing. Vet Microbiol 78, 119-137.

Mutters, R., Ihm, P., Pohl, S., Frederiksen, W. \& Mannheim, W. (1985). Reclassification of the genus Pasteurella Trevisan 1887 on the basis of deoxyribonucleic acid homology, with proposals for the new species Pasteurella dagmatis, Pasteurella canis, Pasteurella stomatis, Pasteurella anatis, and Pasteurella langaa. Int J Syst Bacteriol 35, 309-322.

Olsen, I., Dewhirst, F. E., Paster, B. J. \& Busse, H. J. (2003). Family Pasteurellaceae Pohl 1981, 382VP. In Bergey's Manual of Systematic Bacteriology. Edited by D. J. Brenner, N. R. Krieg, J. T. Staley \& G. M. Garrity. New York: Springer.

Petersen, K. D., Christensen, J. P. \& Bisgaard, M. (1998). Phenotypic and genotypic diversity of organisms previously classified as maltose positive Pasteurella multocida. Zentbl Bakteriol 288, 1-12.

Petersen, K. D., Christensen, H., Bisgaard, M. \& Olsen, J. E. (2001). Genetic diversity of Pasteurella multocida fowl cholera isolates as demonstrated by ribotyping and $16 \mathrm{~S}$ rRNA and partial atpD sequence comparisons. Microbiology 147, 2739-2748.

Rhoades, K. R. \& Rimler, R. B. (1987). Capsular groups of Pasteurella multocida isolated from avian hosts. Avian Dis 31, 895-898.

Rhoades, K. R. \& Rimler, R. B. (1989). Fowl cholera. In Pasteurella and Pasteurellosis, pp. 95-113. Edited by C. F. Adlam \& J. M. Rutter. London: Academic Press.

Rimler, R. B. (2000). Restriction endonuclease analysis using HhaI and HpaII to discriminate among group B Pasteurella multocida associated with haemorrhagic septicaemia. J Med Microbiol 49, $81-87$. 
Rimler, R. B. \& Brogden, K. A. (1986). Pasteurella multocida isolated from rabbits and swine: serologic types and toxin production. Am J Vet Res 47, 730-737.

Rimler, R. B. \& Glisson, J. R. (1997). Fowl cholera. In Diseases of Poultry, pp. 143-159. Edited by B. W. Calnek, H. J. Barnes, C. W. Beard, L. R. McDougald \& Y. M. Saif. Ames: Iowa State University Press.

Rimler, R. B. \& Rhoades, K. R. (1989). Pasteurella multocida. In Pasteurella and Pasteurellosis, pp. 37-73. Edited by C. F. Adlam \& J. M. Rutter. London: Academic Press.

Rimler, R. B., Rhoades, K. R. \& Jones, T. O. (1987). Serological and immunological study of Pasteurella multocida strains that produced septicaemia in fallow deer. Vet Rec 121, 300-301.

Sacchi, C. T., Whitney, A. M., Reeves, M. W., Mayer, L. W. \& Popovic, T. (2002). Sequence diversity of Neisseria meningitidis $16 \mathrm{~S}$ rRNA genes and use of $16 \mathrm{~S}$ rRNA gene sequencing as a molecular subtyping tool. J Clin Microbiol 40, 4520-4527.

Schouls, L. M., Schot, C. S. \& Jacobs, J. A. (2003). Horizontal transfer of segments of the 16S rRNA genes between species of the Streptococcus anginosus group. J Bacteriol 185, 7241-7246.
Snipes, K. P., Hirsh, D. C., Kasten, R. W., Hansen, L. M., Hird, D. W., Carpenter, T. E. \& McCapes, R. H. (1989). Use of an rRNA probe and restriction endonuclease analysis to fingerprint Pasteurella multocida isolated from turkeys and wildlife. J Clin Microbiol 27, 1847-1853.

Watson, P. J. \& Davies, R. L. (2002). Outbreak of Pasteurella multocida septicaemia in neonatal lambs. Vet Rec 151, 420-422.

Westling, K., Bygdeman, S., Engkvist, O. \& Jorup-Ronstrom, C. (2000). Pasteurella multocida infection following cat bites in humans. J Infect 40, 97-98.

Wilson, M. A., Morgan, M. J. \& Barger, G. E. (1993). Comparison of DNA fingerprinting and serotyping for identification of avian Pasteurella multocida isolates. J Clin Microbiol 31, 255-259.

Wilson, M. A., Duncan, R. M., Nordholm, G. E. \& Berlowski, B. M. (1995). Pasteurella multocida isolated from wild birds of North America: a serotype and DNA fingerprint study of isolates from 1978 to 1993. Avian Dis 39, 587-593.

Zhao, G., Pijoan, C., Murtaugh, M. P. \& Molitor, T. W. (1992). Use of restriction endonuclease analysis and ribotyping to study epidemiology of Pasteurella multocida in closed swine herds. Infect Immun 60, 1401-1405. 